Projets

de paysage

\section{Projets de paysage}

Revue scientifique sur la conception et l'aménagement de l'espace

$22 \mid 2020$

Forêt et paysage

\title{
Rêver la forêt, cultiver les arbres
}

Expériences sylvo-artistiques et de plantations agroforestières avec les élèves d'un lycée agricole du Nord de la France

Dreaming of the Forest, Cultivating Trees - Experiments in Tree Art and Agroforestry Plantations with Pupils from an Agricultural Secondary School in Northern France

\section{Alan Guillou}

\section{OpenEdition}

\section{Journals}

\section{Édition électronique}

URL : http://journals.openedition.org/paysage/7698

DOI : 10.4000/paysage.7698

ISSN : 1969-6124

\section{Éditeur :}

École nationale supérieure du paysage de Versailles-Marseille, Institut national des sciences appliquées Centre Val de Loire - École de la nature et du paysage, École nationale supérieure d'architecture et de paysage de Bordeaux, École nationale supérieure d'architecture et de paysage de Lille, Agrocampus Angers

Référence électronique

Alan Guillou, "Rêver la forêt, cultiver les arbres », Projets de paysage [En ligne], 22 | 2020, mis en ligne le 21 juillet 2020, consulté le 24 juillet 2020. URL : http://journals.openedition.org/paysage/7698 DOI : https://doi.org/10.4000/paysage.7698

Ce document a été généré automatiquement le 24 juillet 2020

Projets de paysage 


\section{Rêver la forêt, cultiver les arbres}

Expériences sylvo-artistiques et de plantations agroforestières avec les élèves d'un lycée agricole du Nord de la France

Dreaming of the Forest, Cultivating Trees - Experiments in Tree Art and Agroforestry Plantations with Pupils from an Agricultural Secondary School in Northern France

Alan Guillou

Il était une fois...

un Arbre qui se regardait comme on regarde le sommet inaccessible d'une montagne. De la source profonde jusqu'au sommet touché d'éternité

Il se gravissait à la force de l'imagination.

Il haussait l'Homme au plan de l'Esprit. Et puis, le lien fut rompu, le regard détourné. La magie et le mystère dérobés, cachés sous l'écorce de l'âme humaine. Inconsciente image mythique d'un lien à

l'universel, au sacré, au monde désormais réservé au domaine du rêve.

Les arbres souvent meurent deux fois. Quand la sève les quitte et quand la mémoire ou la légende de leur présence s'efface.

Jamais dans l'histoire de l'humanité notre destin commun n'a été à ce point si incertain... Le Groupe d'experts intergouvernemental sur l'évolution du climat (GIEC) nous a à nouveau instamment rappelé que le temps presse. La perspective désastreuse d'un $+3^{\circ} \mathrm{C},+4^{\circ} \mathrm{C}$ semble toute tracée si, collectivement, nous n'entrons pas dans une phase décisive de passage à l'action! Pour les générations futures, nous nous devons d'engager aujourd'hui la "bataille climatique »... Nous avons dans ce «combat» un 
formidable allié : l'arbre! Cet arbre qui irrigue la pensée humaine depuis l'aube de l'humanité...

2 L'arbre social, l'arbre de la liberté, botanique, de la forêt, l'arbre monde, métaphorique, l'arbre puit de carbone, filtre à particules fines, château d'eau, pourvoyeur de matière organique, protecteur des sols, habitat de nombreuses espèces... amortisseur climatique par excellence !!! Mais, c'est aussi le rendez-vous entre l'individu et l'arbre, et entre les individus eux-mêmes, qui importe aussi dans ce passage à l'acte. L'arbre, comme dénominateur commun, peut nous montrer combien, dans son universalité, il est un élément fédérateur de notre société. Combien par sa grandeur, sa temporalité et sa beauté il est un formidable motif à être et à faire ensemble... pour fonder un avenir qui soit viable. Vivable.

\section{L'ancienne alliance}

3 De nombreuses études sociologiques ont fait la démonstration que nous, humains, nous sommes considérablement éloignés du vaisseau spatial Terre... à en devenir «hors sol »... « extraterrestres ». «L'ancienne alliance est rompue » nous rappelait Jacques Monod (1970, p. 48).

4 Planter un arbre en tant que citoyen c'est d'abord et avant tout réconcilier, renouer un dialogue entre nous et le lieu où nous vivons. C'est aussi, en considérant que nous sommes tous des usagers du paysage, la possibilité donnée à chacun, par l'affirmation du geste, de planter un arbre, de confirmer sa propre responsabilité à l'égard de son environnement de proximité. S'inscrire dans une démarche de chantier nature, de plantation d'arbres, c'est aussi participer à certaine poétique du monde, à son réenchantement... Planter un arbre c'est rentrer en contact avec la matrice de nos existences: le vivant, la nature. C'est en saisir les subtilités, les fragilités et l'importance fondamentale qu'elle revêt. Éprouver son corps dans l'action c'est, enfin, prendre la mesure de sa place dans ce monde...

5 Les structures associatives qui proposent des chantiers nature permettent aux habitants : de créer du lien social ; de mieux comprendre les enjeux environnementaux et de trouver les réponses adaptées ; de restaurer des milieux, des espaces dégradés ; de rendre tangible, concret leur investissement dans l'action; de connaitre et nommer les éléments qui composent leur cadre de vie; de consulter le temps qu'il fait, la saisonnalité; d'amplifier l'instinct de curiosité, notamment chez les plus jeunes, de prendre la mesure de l'érosion de la biodiversité et de ses conséquences directes pour nous; de rendre sa commune plus résiliente, plus résistante aux changements climatiques...

6 Avec un taux de boisement de $9 \%$ contre $28 \%$ pour la moyenne française (Institut forestier national, 2010), les départements du Nord et du Pas-de-Calais sont ceux qui comptent le moins d'arbres en France. Ici, une espèce de fleur disparaît chaque année depuis un siècle. Dix-sept variétés de papillons sur quatre-vingt-dix répertoriées ont disparu en trente ans. Environ 1800 hectares de terres agricoles et d'espaces naturels sont sacrifiés tous les ans au prix de l'étalement urbain. Dans certains secteurs, les sols agricoles soumis à des pratiques culturales intensives connaissent des processus érosifs soutenus qui, sous l'effet de fortes pluies, peuvent engendrer des phénomènes de coulées de boue ${ }^{1}$. 
$7 \quad$ Mais la longue liste des problématiques environnementales de ce territoire ne fait pas oublier que c'est au quotidien que des citoyens agissent à la mesure de leurs moyens pour préserver leur environnement de proximité. À ce titre, l'association Les Planteurs volontaires ${ }^{2}$ mobilise depuis 2013 les habitants souhaitant implanter des arbres dans leur commune.

Parce que les haies bocagères, arbres têtards, vergers et forêts sont des éléments du paysage indispensables aux équilibres de la nature, au bien-être des populations et qu'ils offrent un formidable rempart au changement climatique, il convient de les restaurer, de les protéger et de renforcer leur présence sur le territoire des Hauts-deFrance.

\section{« $\operatorname{Ar}(\mathrm{t}) \mathrm{bres}$ ou la forêt rêvée »}

« $\operatorname{Ar}(t) b r e s$ ou la forêt rêvée » est une exposition collective itinérante et évolutive qui parcourt la nouvelle région des Hauts-de-France depuis $2014^{3}$. Ce projet a été initié par le lycée des sciences de la terre et du vivant d'Hazebrouck, lycée agricole qui forme les agriculteurs de demain. C'est un atelier de pratiques artistiques soutenu par la Drac (direction régionale des Affaires culturelles) depuis septembre 2013 et qui trouve une continuité en 2015 avec le soutien du conseil régional des Hauts-de-France.

Dans le cadre d'un atelier de pratiques artistiques, Laurent Bouquet, professeur d'éducation socioculturelle du lycée agricole, travaille en partenariat avec un artiste associé, Alan Guillou, également cofondateur de l'association Les Planteurs volontaires. Avec eux, les élèves de $2^{\text {de }}$ professionnelle ont élaboré un projet sur le thème de l'arbre et de la forêt : un travail sur la symbolique des représentations de l'arbre et de la forêt dans l'imaginaire collectif, la littérature, les modes de vie, l'art, le cinéma... Chaque promotion, depuis 2013, a bénéficié de $19 \mathrm{~h}$ réparties de la manière suivante $: 6 \mathrm{~h}$ pour évoquer/étudier l'imaginaire sylvestre ; $3 \mathrm{~h}$ pour comprendre le fonctionnement d'un appareil photographique et construire un sténopé; $4 \mathrm{~h}$ d'immersion en forêt (Clairmarais ou Tournehem dans le Pas-de-Calais); $3 \mathrm{~h}$ en demi-groupe pour le tirage des photos en salle obscure/sélection encadrement/scénographie; $3 \mathrm{~h}$ pour l'installation de l'exposition (hôtel de région à Lille, médiathèques...). Ce travail pluridisciplinaire associe également les cours de français, d'anglais, de biologie, de physique, d'agronomie... Recherches, ateliers d'écriture, études de textes littéraires, projections de films ayant comme motif premier ou secondaire l'arbre et la forêt posent les bases d'une culture commune permettant d'appréhender les différentes représentations de la forêt dans l'imaginaire (occidental et oriental). Surtout, il importe dans cet atelier de questionner les élèves, issus du monde agricole, sur la représentation qu'ils se font de la forêt. Dans leur future profession, en plein bouleversement (à la fois économique et agronomique), ces jeunes se doivent de porter un regard neuf sur l'arbre et ses multiples fonctions. Ce projet/atelier vise bien à démontrer toute l'importance que revêt l'image de l'arbre ou de la forêt quand ils sont assumés en tant que symboles, mais également quand ils deviennent un « outil ${ }^{4}$ » au service de l'agriculture et des paysages.

Figure 1. Projet ar(t)bres, sans titre 


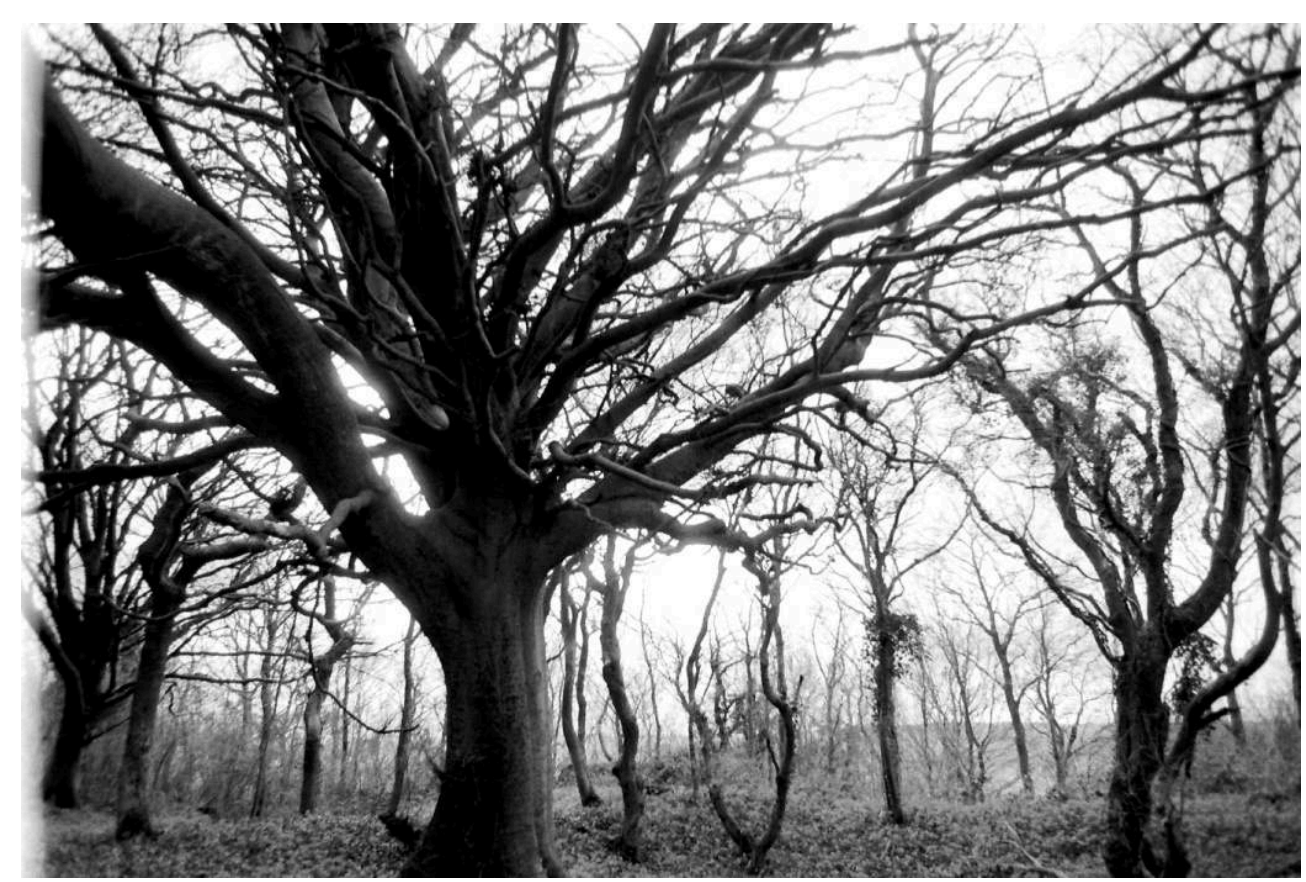

Source : Élèves de 2de professionnelle du lycée agricole des sciences de la terre et du vivant d'Hazebrouck - promotion 2013-2014 - sous la direction d'Alan Guillou.

11 Mais, et c'est bien tout l'enjeu de cet atelier, comment rêver d'arbres quand, historiquement, ils n'ont que très rarement été présents, cultivés, que ce soit sur le parcellaire agricole, dans le paysage de l'exploitation ou même à l'échelle d'une région comme les Hauts-de-France (Dion, 1991)? (On ne prendra pas en compte les particularismes et exceptions que représentent les paysages de l'Avesnois, de la Thiérache...) Comment reconsidérer, réhabiliter la place de l'arbre si, génération après génération, les haies, le bocage, les arbres ne sont tout simplement pas «inscrits", " cultivés » dans la mémoire familiale et paysagère des agriculteurs des grandes plaines du Nord? L'openfield serait alors comme la seule référence paysagère ayant toujours irrigué la pensée des agriculteurs des plaines.

12 À travers ce projet photographique, ces jeunes agriculteurs ont été questionnés quant au regard qu'ils portaient sur l'arbre. L'arbre, la haie sont presque toujours perçus comme une contrainte. La forêt, quant à elle, est davantage appréhendée comme un lieu de loisirs, et ce, à travers la chasse. Mais elle est aussi une surface agricole utile perdue. Jamais, ces éléments boisés du paysage ne sont considérés comme une potentielle ressource.

13 Cet atelier a permis, et souvent avec succès, de développer leur imaginaire sylvestre, de leur permettre de fabriquer des paysages intérieurs avant une hypothétique implantation d'arbres et de forêts sur leur exploitation.

14 À partir de ce travail préalable sur le motif de la forêt et de l'arbre, monde de tous les possibles, la photographie est abordée du point de vue technique et pratique. Des séances de prises de vue en forêt sont programmées avec des sténopés fabriqués par les élèves, avec des appareils anciens, numériques, des smartphones... Ces photos sont " anonymes » car réalisées en atelier collectif ( $1 \mathrm{~h}$ sur les $3 \mathrm{~h}$ passées en forêt). Elles font l'objet d'un travail préparatoire (cadrage, focale, vitesse...) sous la «direction » d'Alan Guillou. 


\section{Des journées entières avec les arbres}

15 Des journées entières avec les arbres, nécessaire immersion prolongée dans la forêt (celles de Tournehem et Clairmarais notamment), confrontent l'élève à lui-même et à sa vision propre de la sylve: incitation à la poésie et au merveilleux pour les uns, confrontation à un univers mystérieux et angoissant pour les autres ; cette immersion a favorisé l'écoute et l'intensité de l'expérience, la possession du sujet et le «corps à corps » dont semblent témoigner certaines épreuves. Cette confrontation physique à l'architecture végétale sera, en classe, partagée autour des références littéraires, picturales, cinématographiques, préalablement relevées par les élèves. Des phrases, des mots, des paroles, des images qui offrent autant de nouvelles pistes d'interprétation à celles et ceux qui parcourront l'exposition, renforçant ainsi l'idée de narration, de récit et de continuité du mythe sylvestre.

Figure 2. Projet ar(t)bres, Sylvain

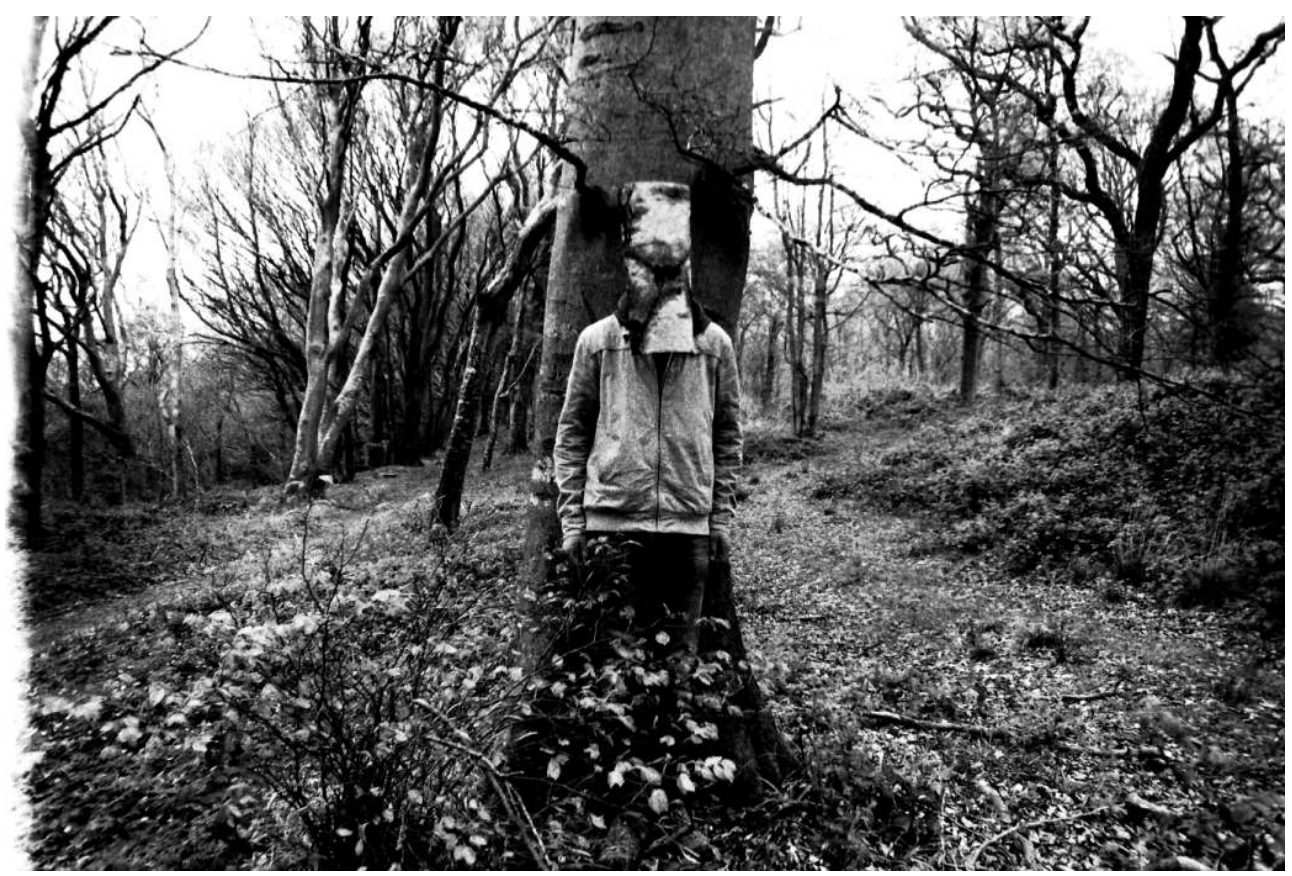

Source : Élèves de $2^{\text {de }}$ professionnelle du lycée agricole des sciences de la terre et du vivant d'Hazebrouck - promotion 2013-2014 - sous la direction d'Alan Guillou.

Lorsqu'elles sont d'origine argentique, certaines photographies sont développées en chambre obscure. Il importe que les élèves comprennent aussi les mécanismes physicochimiques qui sont à l'œuvre lorsque l'on écrit avec la lumière. C'est se poser préalablement la question du choix du noir et blanc. Se demander si l'on peut détourner le réel et la temporalité avec ce type de traitement. C'est pour eux appréhender la sculpturalité des arbres se détachant dans le clair-obscur qui règne au cœur de la forêt. Leur faire comprendre que la photographie ce n'est pas le réel tel quel, ni le sujet même, c'est son ombre, son spectre, son empreinte lumineuse (Bailly, 2008, p. 72-73) : image qui ne pourrait évidemment exister sans l'objet de départ, mais qui s'échappe de lui en le représentant, en le révélant. Pour ce travail créatif, il s'agit là d'interroger leur regard sur les dimensions du "visible et de l'invisible» (MerleauPonty, 2010), l'invisibilité n'étant pas l'opposé du visible, mais plutôt sa doublure... son 
négatif. Démontrer que la visibilité manifeste de la forêt se double d'une visibilité secrète, d'un écho!

Figure 3. Projet ar(t)bres, Chaperonnée

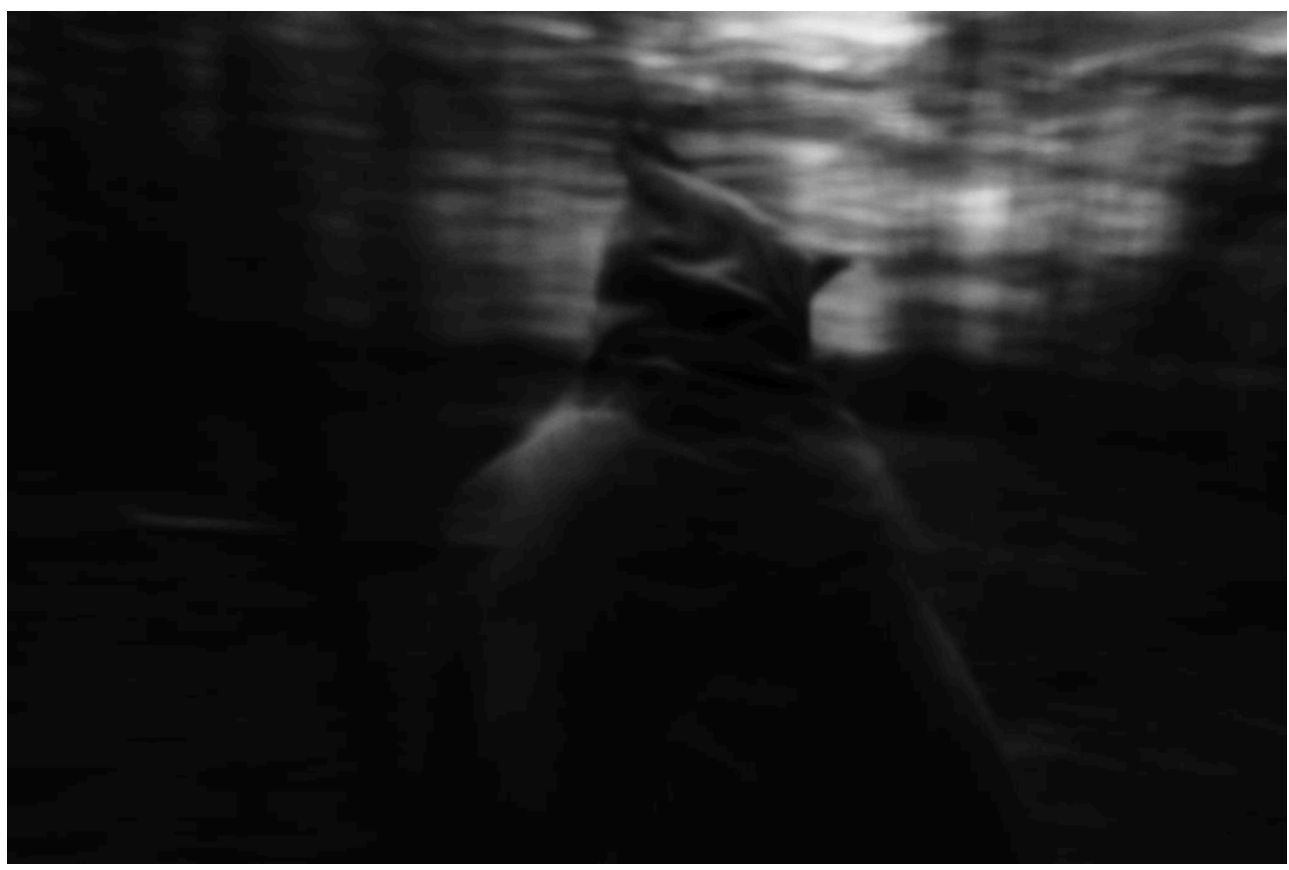

Source : Élèves de $2^{\text {nde }}$ professionnelle du lycée agricole des sciences de la terre et du vivant d'Hazebrouck - promotion 2015-2016 - sous la direction d'Alan Guillou.

17 C'est considérer l'« incident » photographique, le hasard, comme immanent à la prise de vue. C'est exercer son œil graphique, son regard plastique à travers la fenêtre du viseur. C'est aussi prendre la mesure du temps. La durée d'obturation, le nombre limité de prises de vue qui nécessairement imposent des choix, un protocole, une réflexion... À une époque qui a l'image facile, voire compulsive grâce au numérique, rechercher au contraire un certain archaïsme dans le rendu photographique.

Chaque épreuve, renouant parfois avec une poétique artisanale révolue, fait venir à l'esprit l'expression jungienne d' "image primordiale ${ }^{5}$ » et celle, plus naturaliste, de forêt primaire. L'ensemble que recouvrent ces photographies donne parole au réseau des branches, aux murmures des mousses, aux ombres mouvantes... 
Figure 4. Projet ar(t)bres, Doc'Wood

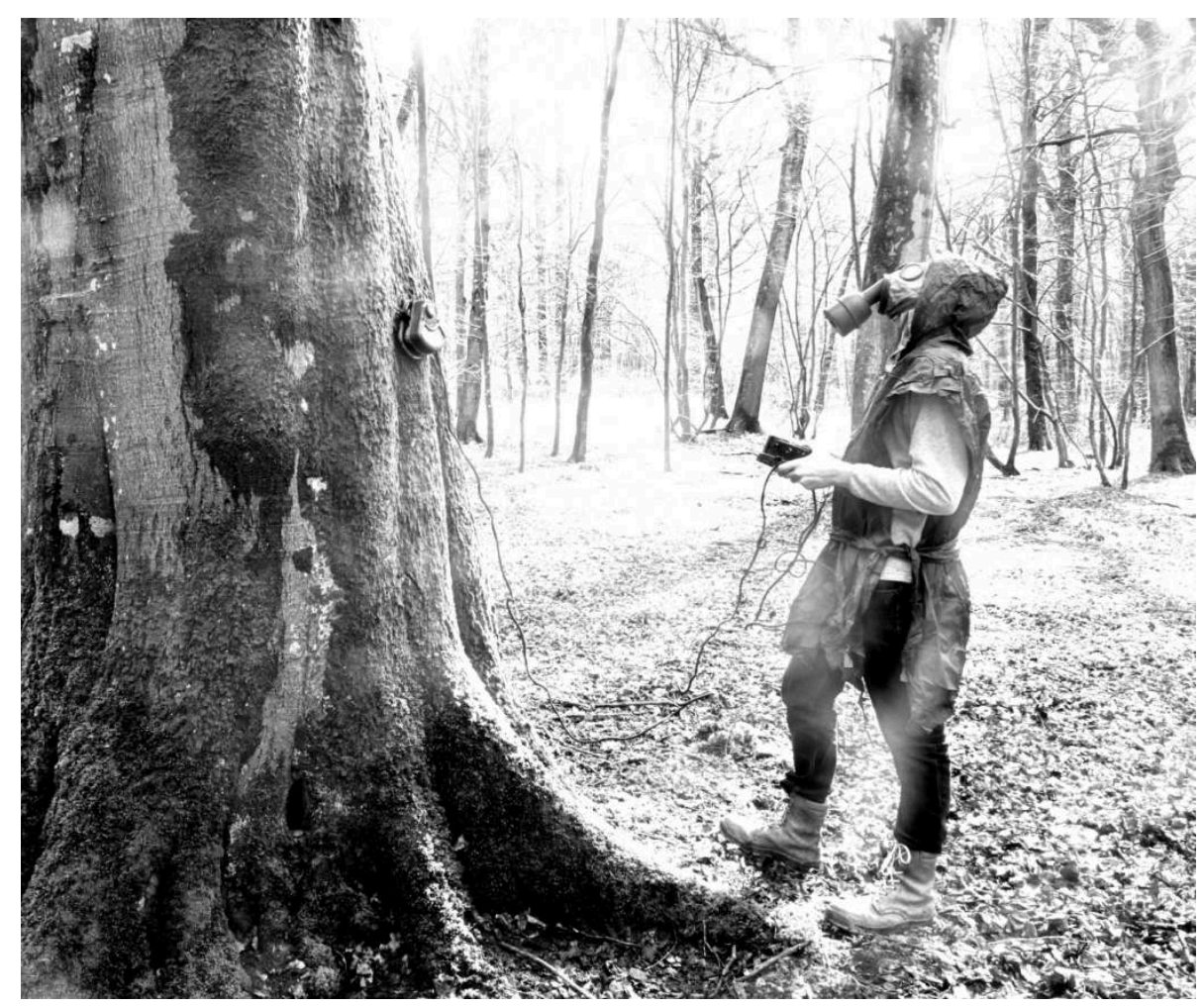

Source : Élèves de $2^{\text {nde }}$ professionnelle du lycée agricole des sciences de la terre et du vivant d'Hazebrouck - promotion 2014-2015 - sous la direction d'Alan Guillou.

Cette forêt, cet arbre métaphorique véhiculent donc encore (mais pour combien de temps ?) des images persistantes d'un paysage mythique que chacun de ces élèves peut percevoir en son fort intérieur : souvenir ténu, primordial et onirique des contes et légendes entendus dans leur enfance, comme une partie de chasse avec leur grandpère, comme la coupe du bois par un matin atone d'hiver, ou une journée à l'école buissonnière. "Les limites du merveilleux" (Maupassant, 1989, p.19) n'ont pas été abolies. Enfouie dans une mémoire cachée, dans cet inconscient collectif, elle est là, la selva oscura (Dante, 2010)! À l'instar de l'esprit humain, elle se révélera parfois ténébreuse, des plus vastes, des plus aventureuses mais aussi des plus riches, sitôt que l'on y pénétrera. Ce souvenir s'adresse mystérieusement à quelques parties de la psyché des élèves... révèle peut-être un rapport renouvelé aux paysages dont ils sont les « maitres d'ouvrage et d'œuvre ».

Chacune de ces images produites en atelier artistique, ramenées du cœur obscur des bois, trouve son propre écho à travers l'actualité. Celle de l'anthropocène. Celle d'une agriculture questionnée sur sa pratique agronomique, technique et paysagère. Après cet atelier, ces jeunes "faiseurs de paysages agricoles» appréhendent-ils l'arbre, le bocage et la forêt comme solution à la crise environnementale et climatique?

21 Aujourd'hui l'exposition « $\operatorname{Ar}(\mathrm{t}) \mathrm{bres}$ » a déjà été installée dans divers lieux culturels de la région (médiathèques, salles d'exposition, hall de l'hôtel de région...). Ces filles et ces garçons qui ne s'étaient jamais considérés comme des artistes, des rêveurs de mondes, sont fiers du travail accompli. 


\section{Projet environnemental et citoyen}

«Ar(t)bres» a également un objectif environnemental et citoyen. Comme précédemment explicité, l'ancienne région du Nord-Pas-de-Calais est l'une des régions les moins boisées de France. Partant de ce constat, le projet a également pour but de sensibiliser à cette problématique environnementale. Des plantations d'arbres sur le territoire sont organisées avec l'association Les Planteurs volontaires, en partenariat avec l'atelier de pratiques environnementales du lycée agricole d'Hazebrouck et les professeurs associés d'agronomie, de techniques culturales. Chaque commune qui accueille l'exposition devient le prétexte à un passage concret à l'action et à une réhabilitation des arbres champêtres.

Les Planteurs volontaires, structure de référence en agroforesterie pour la région des Hauts-de-France (reconnue depuis 2019 par le ministère de l'Agriculture), accompagnent depuis 2013 des groupes d'habitants, des agriculteurs, des communes qui souhaitent développer un programme d'action en faveur des arbres sur des terrains publics et des parcellaires agricoles. Ainsi, en 6 hivers, plus de 6500 " habitants paysagistes » (Lassus, 2000) ont permis la plantation de plus de 111000 arbres dans les Hauts-de-France.

24 En répondant à l'appel de la forêt, en mobilisant leurs parents agriculteurs, leur carnet d'adresses pour trouver les lieux d'implantation de vergers, et de haies, les élèves qui participent à l'atelier engagent pleinement leur profession sur la voie du développement durable et réduisent ainsi un peu plus l'écart entre ce qui a déjà été fait et tout ce qu'il reste encore à accomplir.

Pour ce volet environnemental, les élèves de seconde sont rejoints par d'autres classes de l'institut (première et terminale bac pro, première et terminale bac technologique STAV, BTS...). Des groupes d'étudiants aident à la logistique et à la mise en place des chantiers qui sont participatifs; les habitants, les élèves d'autres établissements scolaires peuvent participer. Les plantations sont réalisées sur les terrains communaux, dans les écoles, les maisons de retraite... ou sur les exploitations agricoles.

Le dernier projet en date, le projet AFRame ${ }^{6}(2018-2021)$, porté par l'Institut supérieur d'agronomie (ISA) de Lille en partenariat avec l'Inra, en partie financé (les arbres) et animé par Les Planteurs volontaires, concerne la mise en place d'un site expérimental d'agroforesterie intraparcellaire sur une parcelle de 18 hectares gérée par la Société civile d'exploitation agricole Dequidt à Ramecourt ${ }^{7}$ (Pas-de-Calais). L'agroforesterie intraparcellaire consiste à aligner des rangées d'arbres à l'intérieur des parcelles agricoles. C'est une pratique agro-écologique permettant d'allier à la fois performance économique et moindre impact sur l'environnement. Comme une grande partie du département est classée en zones vulnérables aux nitrates et que l'aléa érosif des sols reste très fort toute l'année, cette forme d'agroforesterie pourrait bien être une alternative pour une production alimentaire durable et respectueuse du capital sol. 


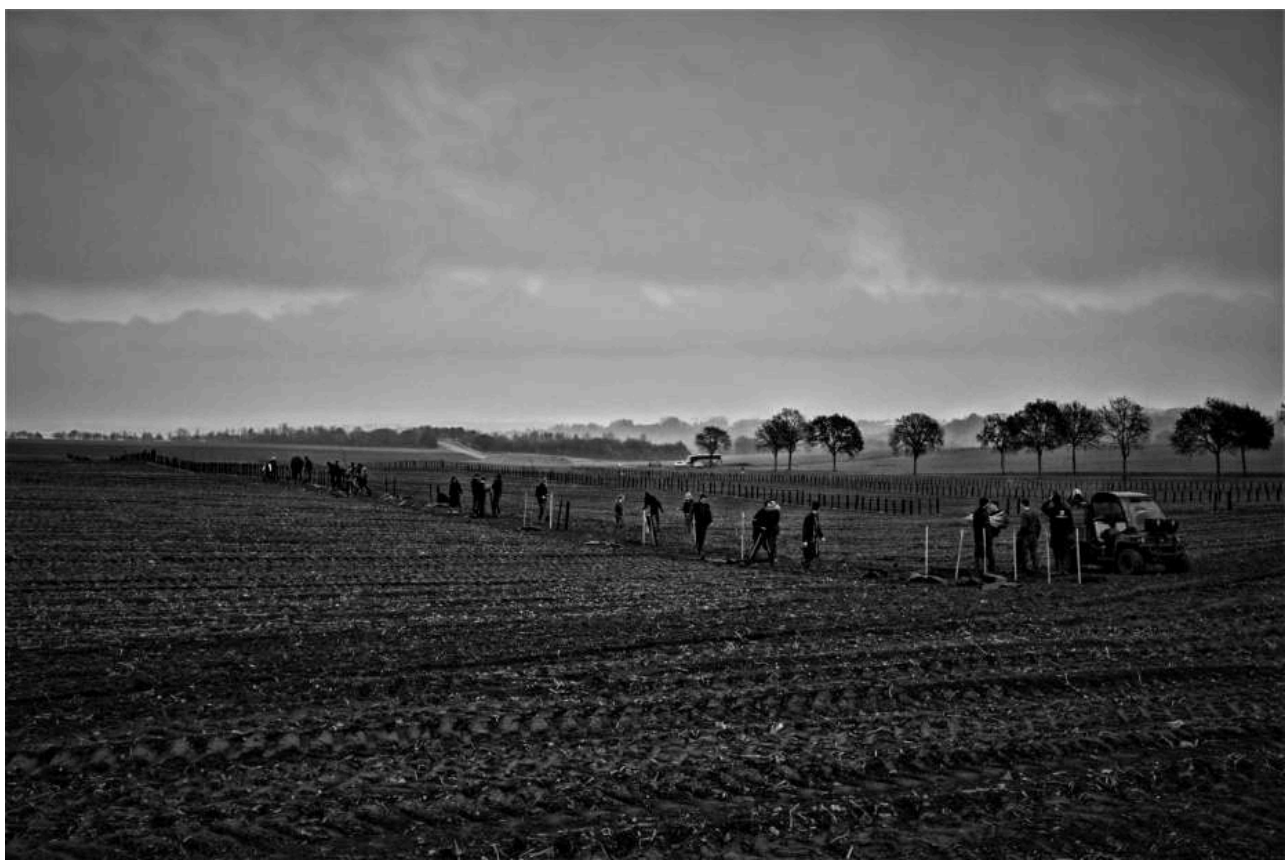

Source : Alan Guillou.

27 Le chantier s'est déroulé du 26 au 30 novembre et le 21 décembre 2018. Il a mobilisé 170 élèves du lycée agricole d'Hazebrouck, une quarantaine d'étudiants et professeurs de l'ISA. Dans des conditions parfois dantesques, avec des pointes de vent à $100 \mathrm{~km} / \mathrm{h}$, les élèves ont permis la plantation de 4438 arbres et arbustes.

Figure 6. Projet AFRame

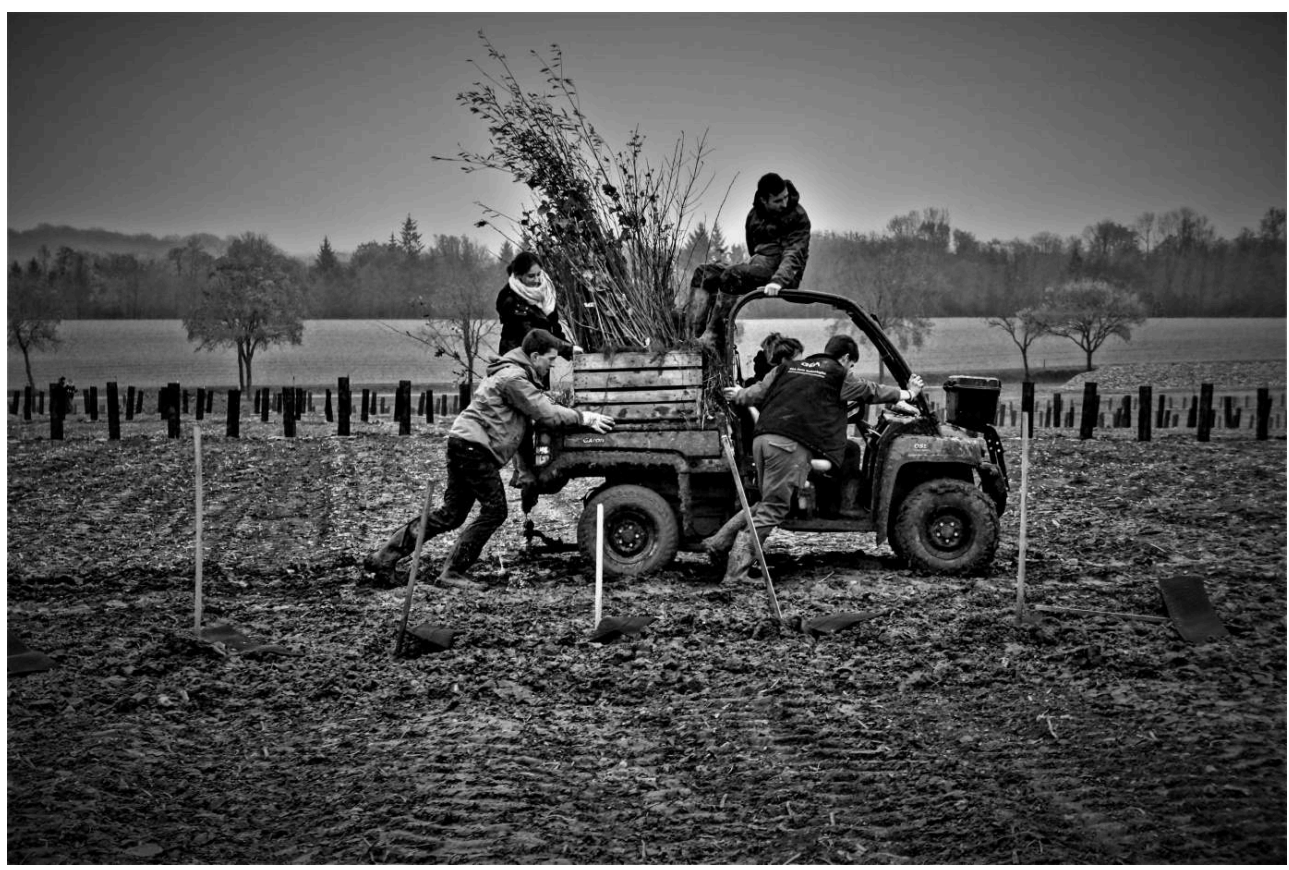

Source : Alan Guillou. 

aux enjeux environnementaux et pour les initier aux multiples services rendus par les arbres. Ce chantier réalisé dans un cadre pédagogique a été l'occasion, pour les professeurs et l'association Les Planteurs volontaires, d'évoquer la biologie des sols, l'eau, les techniques culturales, les auxiliaires de cultures, les continuités écologiques, etc. En rencontrant un agriculteur qui partage une expérience, transmet et explique un cheminement intellectuel, les élèves sont davantage sensibles aux arguments motivant la présence nouvelle de ces arbres sur la surface agricole utile (SAU) du champ. En effet, ce sont bien leurs pairs (pères) qui restent de loin les meilleurs ambassadeurs des projets de reboisement.

En complémentarité avec les réseaux expérimentaux agroforestiers au niveau régional et national, ce dispositif permettra d'évaluer le système dans une région à faible taux annuel d'ensoleillement et avec des cultures spécifiques comme la betterave sucrière et la pomme de terre.

Figure 7. Projet AFRame

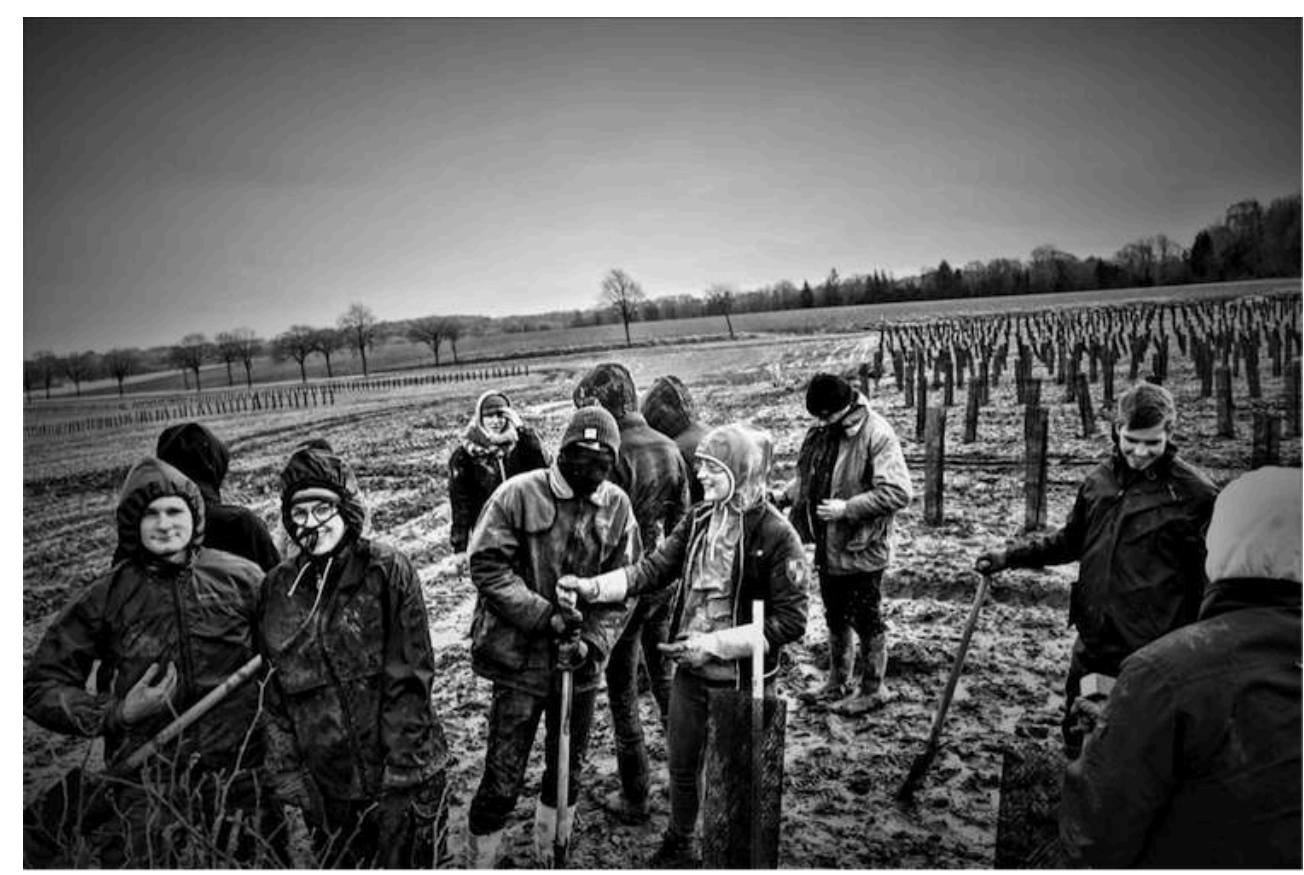

Source : Alan Guillou.

31 Les parcelles agricoles plantées d'arbres seront comparées à des parcelles purement agricoles et forestières. La production de bois et d'énergie, le rendement des cultures, la santé des plantes, le stockage de carbone, le bilan hydrique et azoté, la qualité de l'air, l'érosion, le partage de l'azote entre arbres et cultures, la croissance racinaire, l'évolution de la biodiversité et du paysage, la rentabilité économique et 
l'augmentation du temps de travail à l'échelle de l'exploitation seront estimés et suivis dans le temps par des mesures directes avec des capteurs spécifiques, par modélisation numérique dynamique, avec des protocoles de suivi de la biodiversité et par un observatoire photographique.

Chaque année, des journées thématiques d'information et d'échanges seront organisées avec les agriculteurs et seront suivies par la visite du site.

Figure 8. Projet AFRame

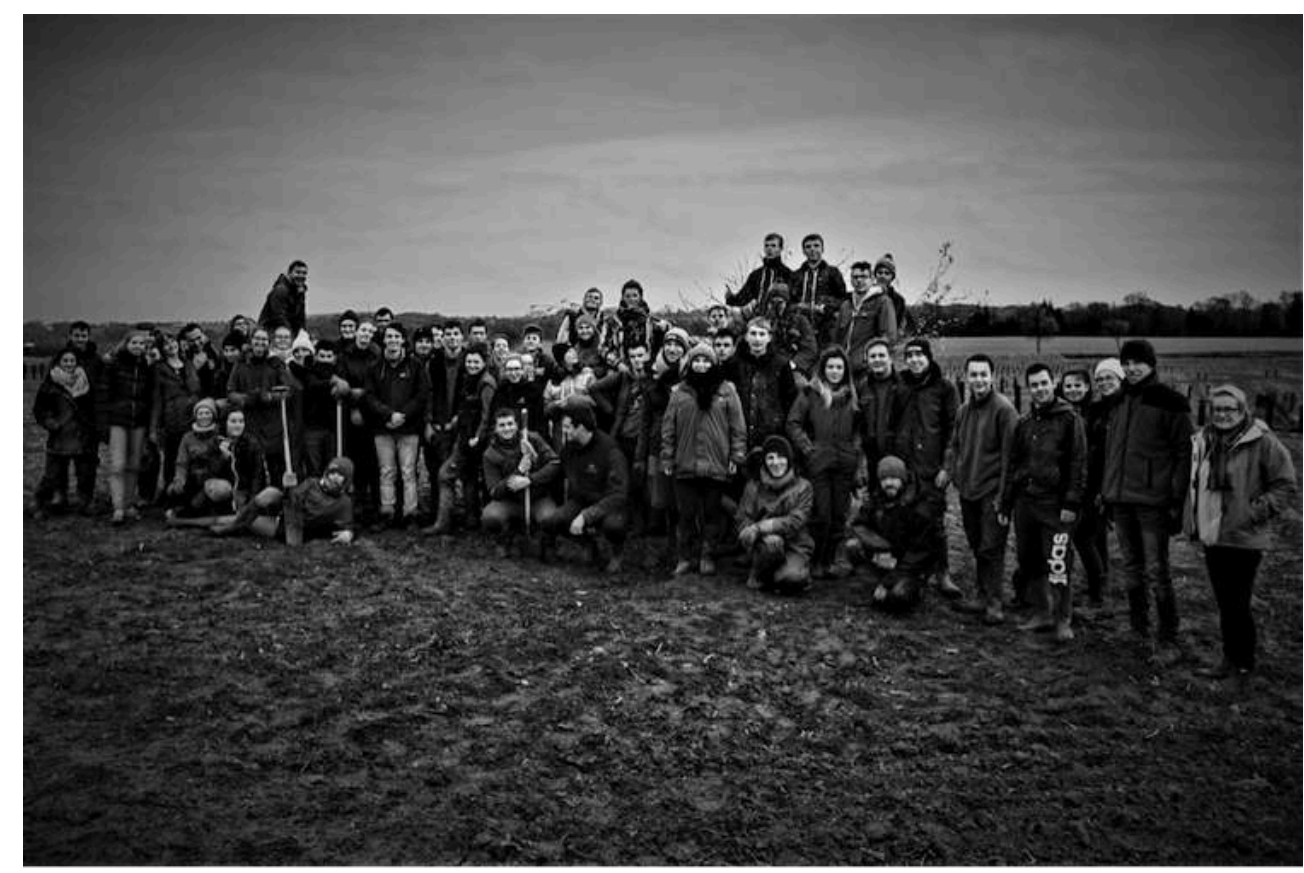

Source : Alan Guillou.

\section{Les tests sur la parcelle}

L'ISA de Lille utilise déjà sur site divers équipements pour le suivi expérimental tels qu'une station météo ou un piézomètre pour évaluer la profondeur de la nappe d'eau et sa circulation dans le temps. Des sondes sont également utilisées pour mesurer l'humidité du sol. Elles permettent d'évaluer l'évolution de la teneur en eau du sol à différentes distances et profondeurs de l'arbre. On peut également observer dans les fosses pédologiques, servant à caractériser le profil du sol, des bougies poreuses pour collecter la solution du sol et mesurer la concentration des eaux en nitrate. 
Figure 9. Projet AFRame, Daniel et ses élèves

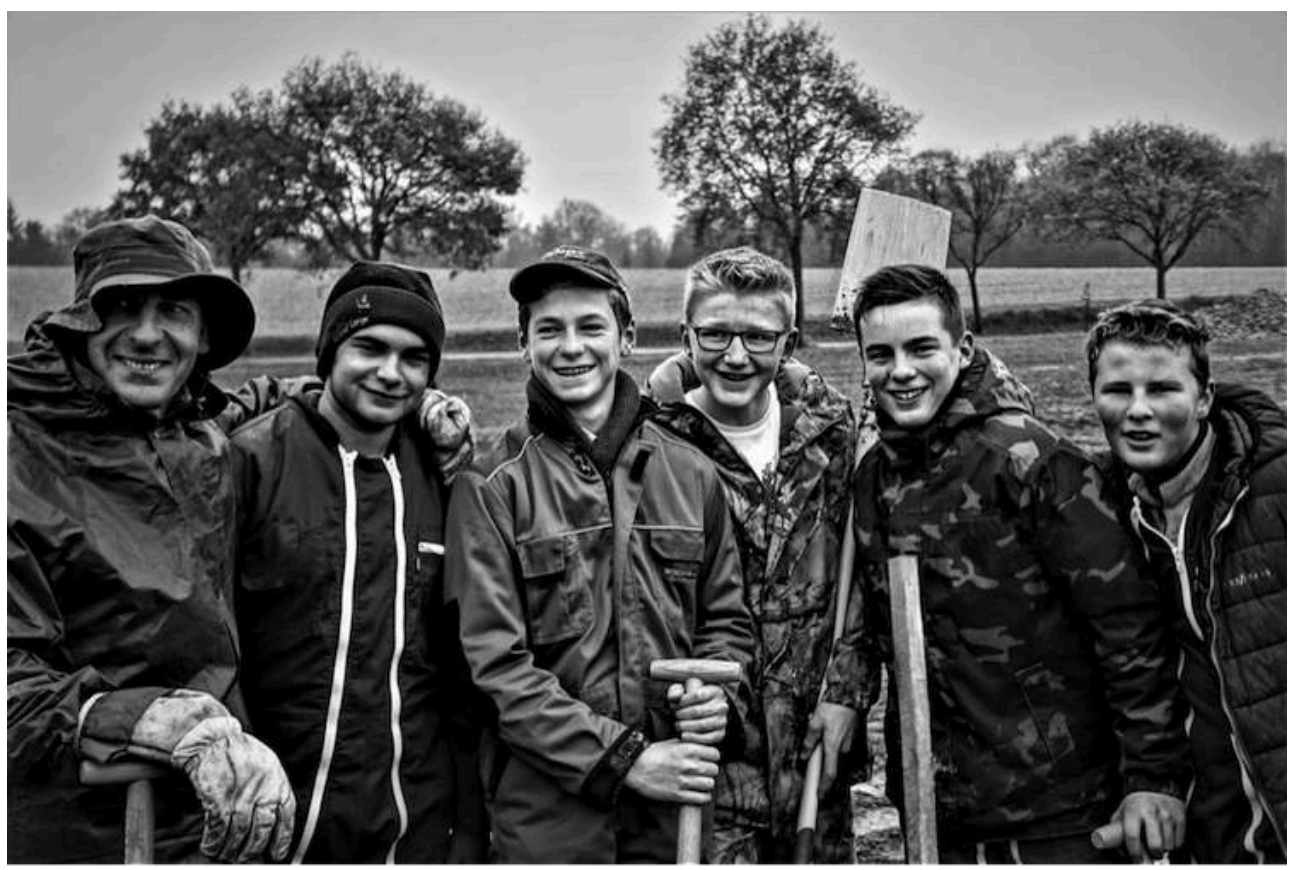

Source : Alan Guillou.

Cette étude permettra également de connaître et de comprendre la croissance racinaire des arbres, l'évolution de la biodiversité aérienne (en partenariat avec Daniel Haubreux, enseignant au lycée agricole, spécialiste des papillons qui, avec ses élèves, a établi un protocole d'observation des papillons) et souterraine, le bilan hydrique et azoté du système, la résilience au changement climatique, l'érosion des sols, la viabilité économique d'un tel système... Ces indicateurs doivent permettre de mieux faire prendre conscience de l'importance des arbres face à la vulnérabilité climatique des champs agricoles. L'ISA a également fait appel à un bulldozer qui a permis de creuser d'immenses fosses et d'y placer des tilleuls adultes afin de pouvoir mesurer la quantité de litière issue de la décomposition des feuilles et branches ainsi que l'ombre que ces arbres portent sur la culture. Après une visite du terrain, on peut aujourd'hui admirer des bandes fleuries parcourant les longues lignes d'arbres et arbustes des témoins forestiers. Des bandes fleuries (phacélie, trèfle, moutarde...) ont favorisé l'arrivée massive des auxiliaires de cultures. Émergeant d'un océan d'orge, les arbres plantés en intraparcellaire s'enracinent désormais durablement dans le paysage.

Dans 8 ans, certains des saules, noisetiers, charmes et prunelliers seront taillés en courte rotation afin d'alimenter en plaquette le marché naissant du bois énergie. Les chênes, châtaigniers, hêtres et tilleuls pousseront encore plus de 50 ans avant d'être à leur tour récoltés pour obtenir des grumes servant à la construction ou à l'ameublement. 


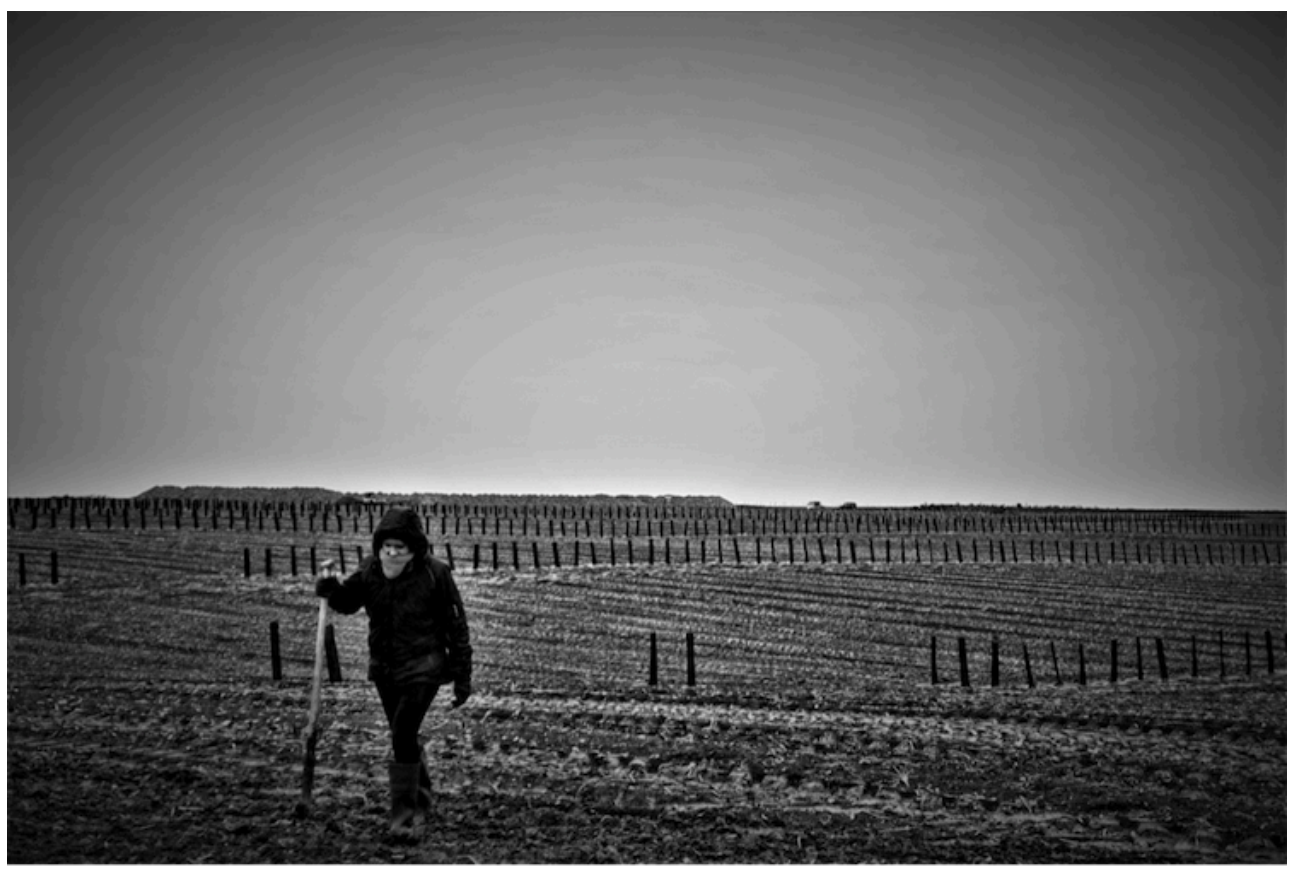

Source : Alan Guillou.

\section{Le temps du rêve}

Prolongement des mythes, des contes et des légendes sylvestres, ces photographies, mais aussi ce chantier qui a pleinement engagé ces jeunes agricultrices et agriculteurs en devenir, expriment et témoignent des désirs, des craintes et des espoirs les plus profonds, des potentialités et des conflits d'un métier en pleine mutation. Regarder leurs photographies, mais aussi ce nouveau paysage, c'est certainement pour eux s'approprier cet espace visionnaire qu'est la forêt, que sont ces arbres. Les élèves du lycée agricole d'Hazebrouck, en participant doublement à cette œuvre collective, permettent à l'homme de continuer à être porteur de mythe ${ }^{8}$, et c'est en stimulant leur imagination et en se mobilisant concrètement en faveur de leur environnement de proximité, qu'ils alimenteront les espoirs d'une époque. Cette écologie symbolique (Descola et Brunois, 2010, p. 379-380 ; Gille, 2014, p. 85-1039) de l'arbre influencera-telle les choix techniques de l'agriculteur en devenir? Permettra-t-elle une prise de conscience? Le projet « $\operatorname{Ar}(\mathrm{t})$ bres", en considérant l'arbre culturel et symbolique comme possible «tête de pont» de pratiques agricoles durables, sert tout à la fois à réhabiliter l'image mythique de la forêt, à réincarner l'âme de l'arbre et à conserver ce que Nietzsche appelait «le sens de la Terre» (1972, p.12). Une vision pour susciter peut-être quelques révélations sur les pratiques des agriculteurs, sinon sur les conséquences d'une nature sacrifiée.

37 Que poussent les arbres et que soit saluée l'énergie créatrice de ces élèves qui, en les plantant, fondent l'avenir. 
Figure 11. Projet AFRame

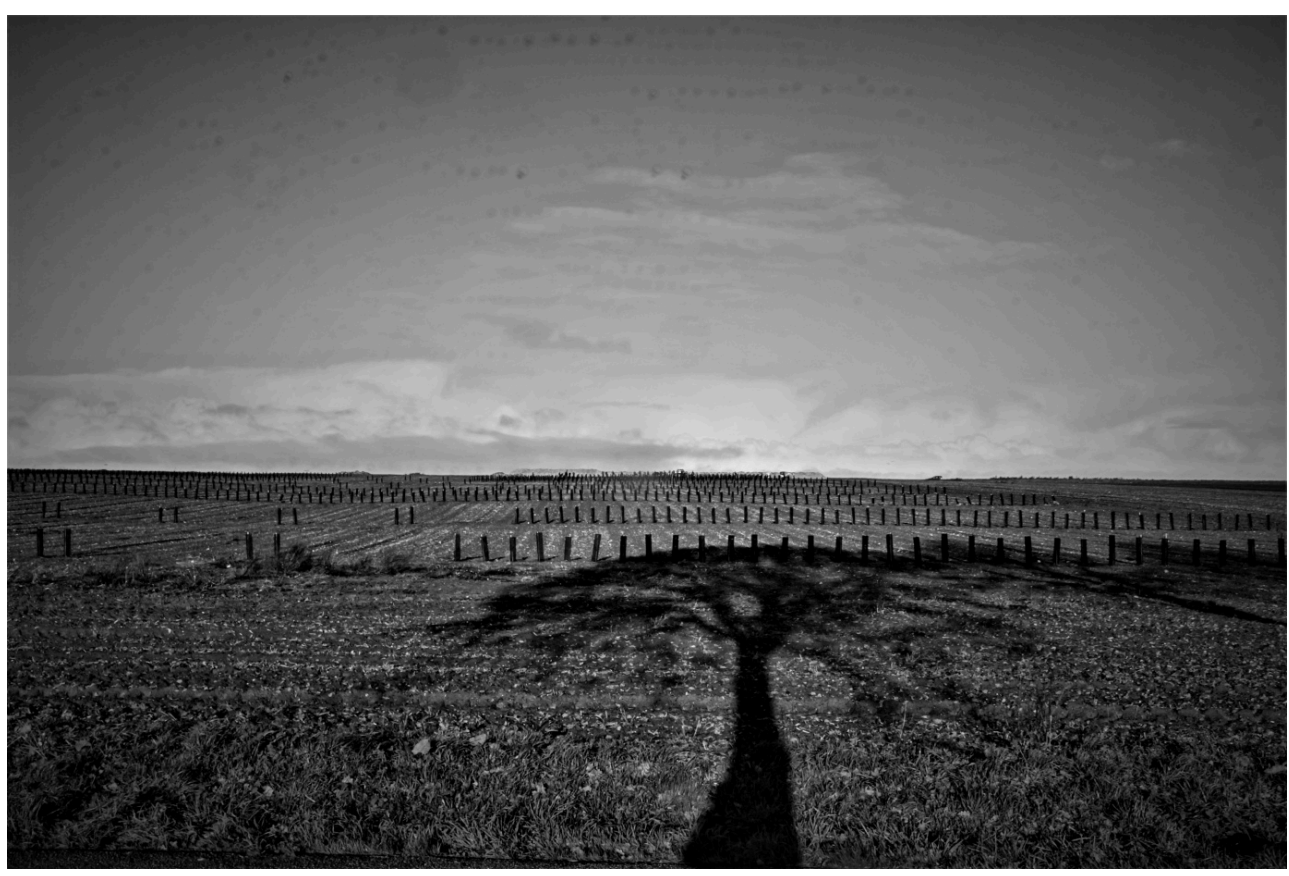

Source : Alan Guillou.

Remerciements à toutes celles et à tous ceux qui fondent l'avenir en plantant des arbres.

\section{BIBLIOGRAPHIE}

Bailly, J..-C., L'Instant et son ombre, Paris, Seuil, 2008.

Dante, La Divine Comédie, Paris, Flammarion, 2010.

Descola, P. et Brunois, F., «Écologie symbolique », Annuaire de l'EHESS, 2010, mis en ligne en juin 2015, URL : https://journals.openedition.org/annuaire-ehess/20035.

Dion, R., Essai sur la formation du paysage rural français, Paris, Flammarion, 1991.

Eliade, M., Mythes, Rêves et Mystères, Paris, Seuil, coll. « Folio essais », 2005.

Gille, B., « De l'écologie symbolique à l'écologie politique. Anthropologie des controverses environnementales chez les Salish côtiers ", Tracés. Revue de Sciences humaines, $\mathrm{n}^{\circ} 22,2012$, mis en ligne en mai 2014, URL : https://journals.openedition.org/traces/5442.

Jung, C. G., Essai d'exploration de l'inconscient, Paris, Seuil, coll. « Folio essais », 2006.

Lassus, B., Jardins imaginaires, Paris, Les Presses de la connaissance, 1977.

Maupassant, G. de, Contes étranges, Paris, Hatier, 1989.

Merleau-Ponty, M., Le Visible et l'Invisible, Paris, Gallimard, 2010. 
Monod, J., Le Hasard et la Nécessité. Essai sur la philosophie naturelle de la biologie moderne, Paris, Seuil, 1970.

Nietzsche, Ainsi parlait Zarathoustra, Paris, Le livre de poche, 1972.

\section{NOTES}

1. https://www.hauts-de-france.developpement-durable.gouv.fr/?L-environnement-en-NordPas-de-Calais-Tome-1-Etat-des-lieux

2. http://planteurs-volontaires.com/

3. https://www.lavoixdunord.fr/82638/article/2016-11-30/artbres-ou-la-foret-revee-allieexposition-et-plantation-citoyenne

4. «Outil» arbre: multifonctionnalité de l'arbre, https://missionbocage.fr/arbre-champetreagriculture-paysage/

5. «Image primordiale » renvoyant à la notion d'archétype de l'arbre, arbre symbolique, motif universel qui irrigue la pensée humaine. Voir Jung, 2006, p. 16.

6. http://www.agroforesterie-hautsdefrance.org/

7. http://www.action-agricole-picarde.com/actualites/agroforesterie-le-projet-experimental-deramecourt:USZV2AXL.html

8. « De ce qui reste encore de mythique dans une existence moderne », voir Eliade, 2005, p. 38-39. 9. «Il est ainsi possible de déterminer, à l'origine de discours écologiques, une autre forme d' écologie symbolique, c'est-à-dire une autre modalité d'organisation des connaissances régissant les relations des humains à la faune et la flore, ainsi que d'autres modalités d'actions permettant une initiative collective en fonction de l'ensemble de ces repères symboliques ».

\section{RÉSUMÉS}

Cette contribution propose le récit d'une expérience artistique et de plantations agroforestières menées par une association auprès d'élèves d'un lycée agricole du Nord de la France. Ce texte souhaite rendre compte de démarches menées dans un cadre pédagogique qui tend à bousculer les cadres de pensée, en insufflant le rôle des arbres et de la forêt auprès d'une profession parfois oublieuse de l'« outil arbre ». En travaillant sur la nature esthétique et symbolique de l'arbre, ces élèves ont marché dans les traces d'un long héritage, d'une longue filiation culturelle de leur métier à la sylve. Ils ont su grâce à l'arbre se projeter dans une temporalité autre, qui n'est plus celle de l'urgence, mais celle de l'introspection. Nécessaire regard à porter au cœur de soi et aux contours d'une profession qui se doit de questionner sa pratique, de réinventer son rapport au lieu, de projeter son destin. C'est l'histoire de cet apprivoisement culturel de l'arbre par le monde agricole que tente de narrer ce texte.

This contribution presents an account of an artistic experiment and agroforestry plantations carried out by an association with the participation of students from an agricultural secondary school in Northern France. The article reports on an educational approach that departs from the usual frames of reference by instilling the importance of the role of trees and forests in a profession that sometimes loses sight of the tree's role as a tool. By working on the aesthetic and 
symbolic aspects of trees, these pupils have adopted an old heritage, a long cultural affiliation of their profession to the forest. Thanks to the tree, they have been able to project themselves into another time frame departing from the notion of urgency to adopt that of introspection. It is necessary to search one's heart and consider the contours of a profession which needs to question its practices, reinvent its relationship to the site and project itself into the future. The article seeks to recount this cultural taming of the tree by the agricultural world.

\section{INDEX}

Keywords : agro-ecology, agriculture, agro-landscape, education, myth

Mots-clés : agroécologie, agriculture, agropaysage, éducation, mythe

\section{AUTEUR}

\section{ALAN GUILLOU}

Alan Guillou, planteur d'arbres devant le Très-Bas, cofondateur et responsable de l'association Les planteurs volontaires.

alanguilloupv[at]gmail[dot]com

http://planteurs-volontaires.com/ 